\title{
LIFT ZONOID ORDER AND FUNCTIONAL INEQUALITIES
}

UDC 519.21

\author{
ALEXEI M. KULIK AND TARAS D. TYMOSHKEVYCH
}

\begin{abstract}
We introduce the notion of a weighted lift zonoid, and we show that the ordering condition imposed on a measure $\mu$ and formulated in terms of the weighted lift zonoids of this measure leads to certain functional inequalities for $\mu$ such as non-linear extensions of Bobkov's shift inequality and weighted inverse log-Sobolev inequality. The choice of the weight $K$ involved in our version of the inverse logSobolev inequality differs substantially from those known in the literature, and it requires that the weight $v$ involved in the definition of the weighted lift zonoid equals the divergence of the weight $K$ with respect to the initial measure $\mu$. We show that such a choice may be useful for proving the direct log-Sobolev inequality as well.
\end{abstract}

\section{INTRODUCTION}

The notions of a zonoid and lift zonoid introduced in [9] have a diverse field of applications. Since the lift zonoid determines the underlying measure uniquely, this concept can be used in multivariate statistics for measuring the variability of laws of random vectors and for ordering these laws; see 10 . The concept of the zonoid equivalence appears to be both naturally motivated by financial applications and useful for proving extensions of the ergodic theorem for zonoid stationary and zonoid swap-invariant random sequences; see [12, 13. Lift zonoids lead naturally to definitions of an associated $\alpha$-trimming and data depth (see [9] and [7]) and to a barycentric representation of points of a space with a given measure (see [9] and [11]).

In this paper, we explore a new field, where the notion of lift zonoid can be applied naturally. As a straightforward extension of the definition of lift zonoid, we introduce a weighted lift zonoid $\hat{Z}^{v}(\mu)$ with a vector-valued weight function $v$. We show that, for properly chosen weights $v$, the ordering condition imposed on a measure $\mu$ and formulated in terms of the weighted lift zonoid of this measure leads to certain functional inequalities for $\mu$ such as non-linear extensions of Bobkov's shift inequality 3 ] and a weighted inverse log-Sobolev inequality. Weighted versions of the classical functional inequalities (Poincaré, log-Sobolev, etc.) have been studied recently in various contexts. The choice of the weight $K$ involved in our version of the inverse log-Sobolev inequality is specific and differs substantially from those known in the literature. This choice is strongly motivated by (an extension of) the functional form of Bobkov's shift inequality, and requires that the weight $v$ involved in the definition of the weighted lift zonoid equals the divergence of the weight $K$ with respect to the initial measure $\mu$. Note that such a choice may be useful for proving the (weighted) direct log-Sobolev inequality as well. In the case of a

2010 Mathematics Subject Classification. Primary 26D10, 39B62, 47D07, 60E15, 60J60.

Key words and phrases. Lift zonoid, weight, shift inequality, log-Sobolev inequality.

The second author was partially supported by the Leonard Euler program, DAAD project \# 55518603 . 
bounded weight, this may lead to new sufficient conditions for the log-Sobolev inequality. We illustrate the range of applications of these conditions in two examples in Section 4

\section{Weighted LIFT ZONOIDS, NON-LINEAR SHIFT INEQUALITIES, AND WEIGHTED INVERSE LOG-SOBOLEV INEQUALITIES}

Let $\mu$ be a probability measure on the Borel $\sigma$-algebra in $\mathbb{R}^{d}$, and let $v: \mathbb{R}^{d} \rightarrow \mathbb{R}^{d}$ be a measurable function such that

$$
\int_{\mathbb{R}^{d}}\|v(x)\| \mu(d x)<\infty .
$$

Here and in what follows we denote by $\|\cdot\|$ the Euclidean norm in $\mathbb{R}^{d}$. We define the weighted zonoid $Z^{v}(\mu)$ with the weight $v$ as the set of all the points in $\mathbb{R}^{d}$ of the form

$$
\int_{\mathbb{R}^{d}} g(x) v(x) \mu(d x)
$$

where $g: \mathbb{R}^{d} \rightarrow[0,1]$ is an arbitrary Borel measurable function. The weighted lift zonoid $\hat{Z}^{v}(\mu)$ is defined as the weighted zonoid of the measure $\delta_{1} \times \mu$ in $\mathbb{R}^{d+1}$. Equivalently, the weighted zonoid $Z^{v}(\mu)$ and the weighted lift zonoid $\hat{Z}^{v}(\mu)$ are the sets of the points of the form

$$
\mathrm{E} g(X) v(X) \in \mathbb{R}^{d} \quad \text { and } \quad(\mathrm{E} g(X), \mathrm{E} g(X) v(X)) \in \mathbb{R}^{d+1},
$$

respectively, where $X$ is a random vector with the distribution $\mu$. This definition is a straightforward generalization of the definitions of the zonoid and lift zonoid (see [10, Definition 2.1]) which corresponds to the case of the function $v(x)=x$.

The lift zonoid $\hat{Z}(\mu)$ is a convex compact set in $\mathbb{R}^{d+1}$, symmetric with respect to the point $\left(\frac{1}{2}, \frac{1}{2} \mathrm{E} X\right)$, which identifies the underlying measure $\mu$ uniquely; see [10]. On the other hand, it can be seen easily that the definition of the weighted lift zonoid $\hat{Z}^{v}(\mu)$ would not change if one restricts the class of Borel measurable functions $g$ within it to the class of the functions of the form

$$
g(x)=G(v(x)), \quad G: \mathbb{R}^{d} \rightarrow[0,1] \text { is Borel measurable. }
$$

This observation leads immediately to the identity $\hat{Z}^{v}(\mu)=\hat{Z}\left(\mu \circ v^{-1}\right)$; that is, the weighted lift zonoid $\hat{Z}^{v}(\mu)$ equals the (usual) lift zonoid of the image of the measure $\mu$ under the mapping $v$. As a corollary, we get that the weighted lift zonoid $\hat{Z}^{v}(\mu)$ is a convex compact set in $\mathbb{R}^{d+1}$, symmetric with respect to the point $\left(\frac{1}{2}, \frac{1}{2} \mathrm{E} v(X)\right)$, and identifies the image measure $\mu \circ v^{-1}$ uniquely.

The following theorem motivates the above definition of the weighted lift zonoid. To formulate it, we need to introduce some notation. Denote by $\gamma_{c}$ the centered Gaussian measure in $\mathbb{R}^{d}$ with the covariance matrix $c^{2} I_{\mathbb{R}^{d}}$. Let

$$
\varphi(x)=\frac{1}{\sqrt{2 \pi}} e^{-x^{2} / 2}, \quad \Phi(x)=\int_{-\infty}^{x} \varphi(y) d y, \quad x \in \mathbb{R},
$$

be the standard Gaussian distribution density function and the standard Gaussian cumulative distribution function, respectively, and let

$$
I(p)=\varphi\left(\Phi^{-1}(p)\right), \quad p \in(0,1), \quad I(0)=I(1)=0,
$$

be the Gaussian isoperimetric function. 
For any measurable $f$ on $\mathbb{R}^{d}$, we write $\mathbf{E}_{\mu} f$ for the integral of $f$ with respect to $\mu$; the function $f$ may be vector-valued, then the integral is understood in the componentwise sense. For a function $f$ taking values in $\mathbb{R}^{+}$, its $\mu$-entropy is defined by

$$
\mathbf{E n t}_{\mu} f=\mathbf{E}_{\mu}(f \log f)-\left(\mathbf{E}_{\mu} f\right) \log \left(\mathbf{E}_{\mu} f\right)
$$

with the convention $0 \log 0=0$.

In what follows, we assume that the measure $\mu$ has the logarithmic gradient $v_{\mu}$; that is, a function $v_{\mu}: \mathbb{R}^{d} \rightarrow \mathbb{R}$ is integrable with respect to $\mu$ and such that

$$
\mathbf{E}_{\mu} \nabla f=-\mathbf{E}_{\mu}\left(v_{\mu} f\right)
$$

for every smooth $f: \mathbb{R}^{d} \rightarrow \mathbb{R}$ with a compact support.

This assumption is equivalent to the following (see [6, Proposition 3.4.3]): there exists the density $p_{\mu}$ of the measure $\mu$ with respect to the Lebesgue measure, which belongs to the Sobolev class $W_{1,1}\left(\mathbb{R}^{d}\right)$; in this case

$$
\left[v_{\mu}\right]_{i}=\frac{\partial_{x_{i}} p_{\mu}}{p_{\mu}}, \quad i=1, \ldots, d .
$$

Theorem 1. I. The following three statements are equivalent.

A. $\widehat{Z}^{v_{\mu}}(\mu) \subset \widehat{Z}\left(\gamma_{c}\right)$.

B. For any smooth function $f: \mathbb{R}^{d} \rightarrow[0,1]$ with a compact support,

$$
\left\|\mathbf{E}_{\mu} \nabla f\right\| \leq c I\left(\mathbf{E}_{\mu} f\right) .
$$

C. For any $h \in \mathbb{R}^{d}$ and $A \in \mathcal{B}\left(\mathbb{R}^{d}\right)$,

$$
\Phi\left(\Phi^{-1}(\mu(A))-c\|h\|\right) \leq \mu(A+h) \leq \Phi\left(\Phi^{-1}(\mu(A))+c\|h\|\right) .
$$

II. Under conditions $\boldsymbol{A}-\boldsymbol{C}$ above, the following inverse log-Sobolev inequality holds:

$$
\left\|\mathbf{E}_{\mu} \nabla f\right\|^{2} \leq 2 c \mathbf{E n t}_{\mu} f \mathbf{E}_{\mu} f
$$

for any smooth function $f: \mathbb{R}^{d} \rightarrow[0, \infty)$ with a compact support.

Remark 1. By definition (see [10, Definition 5.1]), two measures $\mu_{1}$ and $\mu_{2}$ are related by the lift zonoid order (notation: $\mu_{1} \preccurlyeq L Z \mu_{2}$ ) if

$$
\hat{Z}\left(\mu_{1}\right) \subset \hat{Z}\left(\mu_{2}\right) \text {. }
$$

Recall that $\widehat{Z}^{v_{\mu}}(\mu)$ equals the lift zonoid of $\nu_{\mu}:=\mu \circ v_{\mu}^{-1}$. Note that $\nu_{\mu}$ is the distribution of the logarithmic gradient of the measure $\mu$. Hence condition $\mathbf{A}$ can equivalently be formulated as follows: the distribution $\nu_{\mu}$ of the logarithmic gradient of the measure $\mu$ is dominated in the sense of the lift zonoid order by the canonical Gaussian measure in $\mathbb{R}^{d}$.

Theorem [1 is not a genuinely new one. The equivalence of conditions $\mathbf{B}$ and $\mathbf{C}$ is used by S. Bobkov in [3] as a key ingredient of the proof of the shift inequality (6) (in [3], $\mu$ is supposed to be a product-measure but the proof of the equivalence of (5) and (6) does not, in fact, rely on this assumption). The outline of the proof of (7) under (5) and (6) is given in [2]. What we would like to emphasize is that condition $\mathbf{B}$, usually called the functional version of the shift inequality, is equivalent to condition $\mathbf{A}$, which according to Remark 1 can be written as the lift zonoid order relation

$$
\nu_{\mu} \preccurlyeq L Z \gamma_{c} .
$$

It is instructive to compare (8) with the following necessary and sufficient condition for the functional version of the shift inequality to hold, given in $[3]$ in the case where $\mu$ is a 
product-measure with equal marginals $\mu_{1}$. This condition states that there exists $c>0$ such that (5) holds if and only if there exists $\varepsilon>0$ such that

$$
\int_{\mathbb{R}} e^{\varepsilon x^{2}} \nu_{\mu_{1}}(d x) \leq 2
$$

in addition, the optimal constant $c$ in (5) and $\varepsilon$ in (9) are connected by the relation

$$
\frac{1}{\sqrt{6 \varepsilon}} \leq c \leq \frac{4}{\sqrt{\varepsilon}}
$$

For the product measure $\mu(d x)=\prod_{i=1}^{d} \mu_{1}\left(d x_{i}\right)$, the respective distribution of the logarithmic gradient is again a product-measure

$$
\nu_{\mu}(d x)=\prod_{i=1}^{d} \nu_{\mu_{1}}\left(d x_{i}\right),
$$

and in this case, due to [10, Corollary 5.3], (8) is equivalent to

$$
\nu_{\mu_{1}} \preccurlyeq L Z \gamma_{c}^{1} \text {, }
$$

where $\gamma_{c}^{1}$ is the $\mathcal{N}\left(0, c^{2}\right)$-Gaussian measure on $\mathbb{R}$. Both (9) and (11) are conditions on tails of the distribution of the logarithmic gradient of $\mu_{1}$, but (11) is more precise, since it involves the same $c$ as in (5).

The main result of this section, Theorem 2 below, is a generalization of Theorem 1 and is motivated by an observation that the equivalence of conditions $\mathbf{A}$ and $\mathbf{B}$ in Theorem 1 follows in a very straightforward way from the integration-by-parts formula (4); see the proof of Theorem 2 below. With this observation in mind, we introduce a wide class of weights which admit an analogue of the integration-by-parts formula (4). To do that, we recall that the $\mu$-divergence of a function $g: \mathbb{R}^{d} \rightarrow \mathbb{R}^{d}$, if it exists, is defined as the function $\delta_{\mu}(g) \in L_{1}\left(\mathbb{R}^{d}, \mu\right)$ such that

$$
\mathbf{E}_{\mu}(\nabla f, g)_{\mathbb{R}^{d}}=\mathbf{E}_{\mu} f \delta_{\mu}(g)
$$

for every smooth $f: \mathbb{R}^{d} \rightarrow \mathbb{R}$ with a compact support.

The $\mu$-divergence is well defined, for instance, for any $g \in C^{1}$ bounded together with its partial derivatives; in this case,

$$
\delta_{\mu}(g)=-\sum_{i=1}^{d}\left[v_{\mu}\right]_{i} g_{i}-\sum_{i=1}^{d} \partial_{x_{i}} g_{i} .
$$

This follows directly from (4); see [6. Chapter 6] for more detail concerning this subject.

Let a function $v: \mathbb{R}^{d} \rightarrow \mathbb{R}$ be such that

$$
v_{i}=\delta_{\mu}\left(K_{i}\right), \quad i=1, \ldots, d,
$$

for some function $K$ taking values in the space of $d \times d$-matrices, where $K_{i}$ denotes the $i$-th row of the matrix $K$. Then

$$
\mathbf{E}_{\mu}(K \nabla f)=\mathbf{E}_{\mu} f v
$$

for every smooth $f$ with a compact support; here and below we treat elements of $\mathbb{R}^{d}$ as vector-columns. Formula (13) is a straightforward extension of the integration-by-parts formula (4), where the gradient $\nabla$ is replaced by the weighted gradient $K \nabla$ with the matrix-valued weight $K$, and the logarithmic gradient $v_{\mu}$ is replaced by the $\mu$-divergence of $K$. Furthermore, if $K$ satisfies some extra regularity condition, e.g.,

$$
K: \mathbb{R}^{d} \rightarrow \mathbb{R}^{d \times d} \quad \text { is Lipschitz, }
$$


then for every $h \in \mathbb{R}^{d}$ there exists a flow of solutions $\left\{\Psi_{t}^{K, h}(x), t \in \mathbb{R}, x \in \mathbb{R}^{d}\right\}$ of the Cauchy problem

$$
d \Psi_{t}(x)=\left(K^{*} h\right)\left(\Psi_{t}(x)\right) d t, \quad \Psi_{0}(x)=x .
$$

Theorem 2. I. Let $v=\left(v_{i}\right)_{i=1}^{d}$ satisfy (12). Then the following two statements are equivalent.

A1. $\widehat{Z}^{v}(\mu) \subset \widehat{Z}\left(\gamma_{c}\right)$.

B1. For any smooth function $f: \mathbb{R}^{d} \rightarrow[0,1]$ with a compact support,

$$
\left\|\mathbf{E}_{\mu} K \nabla f\right\| \leq c I\left(\mathbf{E}_{\mu} f\right) .
$$

If, in addition, the matrix-valued function $K$ satisfies (14), then $\boldsymbol{A} \mathbf{1}$ and $\boldsymbol{B} \mathbf{1}$ are equivalent to the following.

C1. For any $h \in \mathbb{R}^{d}$ and $A \in \mathcal{B}\left(\mathbb{R}^{d}\right)$,

$$
\Phi\left(\Phi^{-1}(\mu(A))-c\|h\|\right) \leq \mu\left(\left[\Psi_{1}^{K, h}\right]^{-1}(A)\right) \leq \Phi\left(\Phi^{-1}(\mu(A))+c\|h\|\right) .
$$

II. Under condition $\boldsymbol{A 1}$, or equivalently $\mathbf{B 1}$, the following weighted inverse log-Sobolev inequality holds:

$$
\left\|\mathbf{E}_{\mu} K \nabla f\right\|^{2} \leq 2 c^{2} \mathbf{E n t}_{\mu} f \mathbf{E}_{\mu} f
$$

for any smooth function $f: \mathbb{R}^{d} \rightarrow[0, \infty)$ with a compact support.

Note that condition $\mathbf{A} \mathbf{1}$ is just the lift zonoid order relation for the image measure of $\mu$ under $v$ :

$$
\mu \circ v^{-1} \preccurlyeq L Z \gamma_{c} .
$$

Prior to giving the proof of Theorem 2, we summarize. The lift zonoid order condition (8) is a criterion for the shift inequality written either in its explicit form (6) or in its functional form (5). This equivalence is rather flexible in the following sense: if the logarithmic gradient $v_{\mu}$ in (8) is replaced by another weight $v$ of the form

$$
v=\delta_{\mu}(K)
$$

(see (12)), then respective lift zonoid order condition (19) is still equivalent to the weighted version (16) of the functional form of a (generalized) shift inequality. The explicit form of the (generalized) shift inequality in that case is available as well, and concerns, instead of linear shifts, the transformations of the initial measure $\mu$ by the flows of solutions to (15).

Proof of Theorem 2: Statement I. The lift zonoid $\hat{Z}(\gamma)$ of a standard Gaussian measure $\gamma$ in $\mathbb{R}^{d}$ can be identified in the following way: for a given $\alpha \in(0,1)$, the projection on the last $d$ coordinates of the section of $\hat{Z}(\gamma)$ by the hyperplane $\{\alpha\} \times \mathbb{R}^{d}$ coincides with the ball centered at 0 and having the radius $I(\alpha)$; see [9, Section 6.3] or [11, Proposition 3.4]. It is easy to see from the definition of the lift zonoid that

$$
\hat{Z}\left(\gamma_{c}\right)=c \hat{Z}(\gamma)
$$

Hence condition A1 can be written equivalently as follows: for every Borel measurable $g: \mathbb{R}^{d} \rightarrow[0,1]$ such that $\mathbf{E}_{\mu} g=\alpha$,

$$
\left\|\mathbf{E}_{\mu}(g v)\right\| \leq c I(\alpha)=c I\left(\mathbf{E}_{\mu} g\right) .
$$


By the standard approximation argument, the above condition is equivalent to a similar one with a Borel measurable function $g$ replaced by a smooth and compactly supported $f$. Since, for such $f$,

$$
\left\|\mathbf{E}_{\mu}(f v)\right\|=\left\|\mathbf{E}_{\mu}(K \nabla f)\right\|
$$

by (13), conditions $\mathbf{A} \mathbf{1}$ and $\mathbf{B} 1$ are equivalent.

The proof of the equivalence of $\mathbf{B} \mathbf{1}$ and $\mathbf{C} 1$ follows the same lines as in the S. Bobkov proof in [3] for the case of product measures and linear shifts. To make the exposition self-sufficient, we expose the key steps of this proof.

Put $R_{r}(p)=\Phi\left(\Phi^{-1}(p)+r\right), r \geq 0, p \in(0,1)$. Then

- for every $r \geq 0$, the function $R_{r}$ is concave;

- the family $\left\{R_{r}, r \geq 0\right\}$ is a semigroup with respect to the composition of the functions, i.e.,

$$
R_{r_{1}} \circ R_{r_{2}}=R_{r_{1}+r_{2}}
$$

- the function $R_{0}$ is an identity, and the "generator" of the semigroup $\left\{R_{r}, r \geq 0\right\}$ equals the Gaussian isoperimetric function $I$ in the sense that

$$
\frac{R_{r}(p)-p}{r} \rightarrow I(p), \quad r \rightarrow 0+
$$

Similarly, the family of functions $S_{r}(p)=\Phi\left(\Phi^{-1}(p)-r\right), r \geq 0, p \in(0,1)$ has the following properties:

- for every $r \geq 0$, the function $S_{r}$ is convex;

- the family $\left\{S_{r}, r \geq 0\right\}$ is a semigroup with respect to the composition of the functions;

- the function $S_{0}$ is an identity, and the generator of the semigroup $\left\{S_{r}, r \geq 0\right\}$ equals $(-I)$.

Observe that $\mathbf{C} 1$ is equivalent to the following.

C2. For any $h \in \mathbb{R}^{d}$ and Borel measurable $f: \mathbb{R}^{d} \rightarrow[0,1]$,

$$
S_{c\|h\|}\left(\mathbf{E}_{\mu} f\right) \leq \mathbf{E}_{\mu}\left(f \circ \Psi_{1}^{K, h}\right) \leq R_{c\|h\|}\left(\mathbf{E}_{\mu} f\right) .
$$

Indeed, taking $f=\mathbb{1}_{A}$, we get $\mathbf{C} 1$ from $\mathbf{C 2}$. Inversely, under $\mathbf{C} 1$ by the concavity of $R_{r}$ and Jensen's inequality, we have

$$
\begin{aligned}
\mathbf{E}_{\mu}\left(f \circ \Psi_{1}^{K, h}\right) & =\int_{0}^{\infty} \mu\left(\left\{x: f\left(\Psi_{1}^{K, h}(x)\right) \geq t\right\}\right) d t \leq \int_{0}^{\infty} R_{c\|h\|}(\mu(\{x: f(x) \geq t\})) d t \\
& \leq R_{c\|h\|}\left(\int_{0}^{\infty} \mu(\{x: f(x) \geq t\}) d t\right)=R_{c\|h\|}\left(\mathbf{E}_{\mu} f\right) .
\end{aligned}
$$

The proof of the left-hand side inequality in (21) is similar and omitted. Hence C1 and $\mathbf{C 2}$ are equivalent.

To get $\mathbf{B 1}$ from $\mathbf{C 2}$, take $t h$ instead of $h$ and differentiate the right-hand side of (21) with respect to $t$ at the point $t=0$. In more detail, denote $f_{t}(x)=f\left(\Psi_{1}^{K, t h}(x)\right)$. Then

$$
f_{t}(x)=f\left(\Psi_{t}^{K, h}(x)\right)
$$

and therefore there exits a continuous derivative

$$
\partial_{t} f_{t}(x)=\left((\nabla f)\left(\Psi_{t}^{K, h}(x)\right),\left(K^{*} h\right)\left(\Psi_{t}^{K, h}(x)\right)\right)_{\mathbb{R}^{d}} .
$$

Since $f$ is smooth and compactly supported and $K$ satisfies (14), this derivative is bounded as a function of $(t, x) \in[0, T] \times \mathbb{R}^{d}$ for every fixed $T$. Therefore by the dominated 
convergence theorem

$$
\frac{1}{t}\left(\mathbf{E}_{\mu} f_{t}-\mathbf{E}_{\mu} f\right) \rightarrow \mathbf{E}_{\mu}\left(\nabla f, K^{*} h\right)_{\mathbb{R}^{d}}=\left(\mathbf{E}_{\mu} K \nabla f, h\right)_{\mathbb{R}^{d}}, \quad t \rightarrow 0+.
$$

Now

and we get from (21)

$$
\frac{1}{t}\left(R_{c t\|h\|}\left(\mathbf{E}_{\mu} f\right)-\mathbf{E}_{\mu} f\right)=c\|h\| I\left(\mathbf{E}_{\mu} f\right)
$$

$$
\left(\mathbf{E}_{\mu} K \nabla f, h\right)_{\mathbb{R}^{d}} \leq c\|h\| I\left(\mathbf{E}_{\mu} f\right), \quad h \in \mathbb{R}^{d} .
$$

Taking sup over all $h$ with $\|h\|=1$, we get (16).

To get $\mathbf{C 2}$ from $\mathbf{B} 1$, consider first the case where $f$ is smooth and compactly supported and such that $0<\mathbf{E}_{\mu} f<1$. By (16), for a given $h \in \mathbb{R}^{d}$ we have that

Recall that

$$
\left.\frac{d}{d t}\right|_{t=0} \mathbf{E}_{\mu} f_{t}=\left(\mathbf{E}_{\mu} K \nabla f, h\right)_{\mathbb{R}^{d}} \leq c\|h\| I\left(\mathbf{E}_{\mu} f\right) .
$$

$$
\left.\frac{d}{d t}\right|_{t=0} R_{c\|h\| t}\left(\mathbf{E}_{\mu} f\right)=c\|h\| I\left(\mathbf{E}_{\mu} f\right) .
$$

Therefore, for every $\varrho>1$, there exists $\delta=\delta(f)>0$ such that

$$
\mathbf{E}_{\mu} f_{t} \leq R_{\varrho c\|h\| t}\left(\mathbf{E}_{\mu} f\right)
$$

for every $t \in(0, \delta)$. Note that if $t_{1} \in(0, \delta(f))$ and $t_{2} \in\left(0, \delta\left(f_{t_{1}}\right)\right)$, then

$$
\mathbf{E}_{\mu} f_{t_{1}+t_{2}}=\mathbf{E}_{\mu}\left(f_{t_{1}} \circ \Psi_{t_{2}}^{K, h}\right) \leq R_{\varrho c\|h\| t_{1}}\left(\mathbf{E}_{\mu} f_{t_{1}}\right) \leq R_{\varrho c\|h\|\left(t_{1}+t_{2}\right)}\left(\mathbf{E}_{\mu} f\right) ;
$$

here we have used the flow property of $\left\{\Psi_{t}^{K, h}, t \in \mathbb{R}\right\}$, the semigroup property of $\left\{R_{r}, r \geq 0\right\}$, and monotonicity of $R_{r}$. Since the derivative $\partial_{t} f_{t}$ is uniformly continuous with respect to $(t, x) \in[0, T] \times \mathbb{R}^{d}$ for every fixed $T$, it can be shown that

$$
\delta_{T}=\inf _{t \in[0, T]} \delta\left(f_{t}\right)>0 .
$$

Then, applying (23) at most $T / \delta_{T}$ times, we get that (22) holds for every $t \in[0, T]$. Consequently, (22) holds for every $t \in \mathbb{R}^{+}$and $\varrho$ therein can be replaced by 1 . This gives the right-hand side inequality in (21) for smooth and compactly supported functions $f$ such that $0<\mathbf{E}_{\mu} f<1$. By an approximation argument, this can be extended to any measurable $f: \mathbb{R}^{d} \rightarrow[0,1]$. The proof of the left-hand side inequality in (21) is completely analogous and omitted.

Proof of Theorem 2;: Statement II. The following lemma is a straightforward extension of a part of Proposition 2 in 2] (the one which states the equivalence of $P_{1}(c)$ and $P_{2}(c)$ in the notation of [2]).

Lemma 1. Statement $\boldsymbol{B} \mathbf{1}$ is equivalent to the following.

B2: For any smooth function $f: \mathbb{R}^{d} \rightarrow[0,1]$ with a compact support,

$$
\sqrt{\left(\mathbf{E}_{\mu} I(f)\right)^{2}+\frac{1}{c^{2}}\left\|\mathbf{E}_{\mu} K \nabla f\right\|^{2}} \leq I\left(\mathbf{E}_{\mu} f\right) .
$$

The proof is completely analogous to that from [2]; therefore, we just sketch it. The implication $\mathbf{B 2} \Rightarrow \mathbf{B} \mathbf{1}$ is trivial. To get the inverse implication, recall first that the standard Gaussian measure $\gamma^{d}$ on $\mathbb{R}^{d}$ satisfies B2 with $c=1$ and identity matrix $K$; see [2, Section 2]. Consider a smooth function $f: \mathbb{R}^{d} \rightarrow[0,1]$ with a compact support, and let $F(r)=\mu(\{x: f(x) \leq r\})$ be its distribution function with respect to $\mu$. Assume 
that $F$ is absolutely continuous with respect to the Lebesgue measure on $\mathbb{R}$, and choose $r \in \mathbb{R}$ and $\varepsilon>0$. Define

$$
\psi_{\varepsilon}(x)=\mathbb{I}_{[0, r]}(x)+\left(1-\frac{x-r}{\varepsilon}\right) \mathbb{I}_{[r, r+\varepsilon]}(x) .
$$

Applying $\mathbf{B} 1$ to the function $g=\psi_{\varepsilon}(f)$ and letting $\varepsilon \rightarrow 0$, we get

$$
F^{\prime}(r)\|\theta(r)\| \leq c I(F(r)) \text { for } \mu \circ f^{-1} \text {-a.a. } r \in \mathbb{R},
$$

where $\theta(r)=\mathbf{E}_{\mu}(K \nabla f \mid f=r)$. Denote $k=F^{-1} \circ \Phi$, then $k$ transforms the standard Gaussian measure $\gamma^{1}$ on $\mathbb{R}$ to $\mu \circ f^{-1}$. Taking the derivative in the identity $F(k)=\Phi$, we get $k^{\prime} F^{\prime}(k)=\varphi$. Then from (25) with $r=k(x)$, we obtain

$$
\frac{1}{c}\|\theta(k(x))\| \leq k^{\prime}(x)
$$

almost surely with respect to $\gamma^{1}$. We have already mentioned that a standard Gaussian measure satisfies B2 with $c=1$ and the identity matrix $K$; for the case $d=1$ this can be written as

$$
\sqrt{\left(\int_{\mathbb{R}} I(g) d \gamma^{1}\right)^{2}+\left(\int_{\mathbb{R}} g^{\prime} d \gamma^{1}\right)^{2}} \leq I\left(\int_{\mathbb{R}} g d \gamma^{1}\right) .
$$

Applying this inequality to $g=k$ and using (26) we get

$$
\sqrt{\left(\int_{0}^{1} I(r) d F(r)\right)^{2}+\left(\int_{0}^{1} \frac{1}{c}\|\theta(r)\| d F(r)\right)^{2}} \leq I\left(\int_{0}^{1} r d F(r)\right) .
$$

Here we took into account that the image of $\gamma^{1}$ under $k$ is $\mu \circ f^{-1}$, and $\mu \circ f^{-1}$ is supported in $[0,1]$. Using the inequality

$$
\int_{0}^{1}\|\theta(r)\| d F(r) \geq\left\|\int_{0}^{1} \theta(r) d F(r)\right\|=\left\|\mathbf{E}_{\mu} K \nabla f\right\|,
$$

we complete the proof of the required statement. The additional assumption that $\mu \circ f^{-1}$ is absolutely continuous can be removed by an approximation argument.

According to Lemma 1, statement II of Theorem 2 follows if one shows that B2 implies (18) for any non-negative smooth compactly supported function $f$. Choose $\varepsilon$ small so that $\varepsilon f$ assumes values in $[0,1]$ and one can apply B2. After trivial transformations, we get

$$
\frac{1}{c^{2}}\left\|\mathbf{E}_{\mu} K \nabla f\right\|^{2} \leq \frac{I^{2}\left(\varepsilon \mathbf{E}_{\mu} f\right)-\left(\mathbf{E}_{\mu} I(\varepsilon f)\right)^{2}}{\varepsilon^{2}} .
$$

Hence the required statement follows from the relation

$$
\lim _{\varepsilon \rightarrow 0+} \frac{I^{2}\left(\varepsilon \mathbf{E}_{\mu} f\right)-\left(\mathbf{E}_{\mu} I(\varepsilon f)\right)^{2}}{\varepsilon^{2}}=2 \mathbf{E n t}_{\mu} f \mathbf{E}_{\mu} f .
$$

The latter relation can be proved straightforwardly using the asymptotic expansion

$$
I(\varepsilon)=\varepsilon \sqrt{2 \log \frac{1}{\varepsilon}}-\frac{\varepsilon \log \left(2 \log \frac{1}{\varepsilon}\right)}{2 \sqrt{2 \log \frac{1}{\varepsilon}}}+\frac{\varepsilon}{\sqrt{2 \log \frac{1}{\varepsilon}}}+\frac{\varepsilon \kappa(\varepsilon)}{\sqrt{2 \log \frac{1}{\varepsilon}}},
$$

where $\kappa(\varepsilon) \rightarrow 0$ as $\varepsilon \rightarrow 0+$; the detailed exposition is straightforward but cumbersome and therefore is omitted.

Asymptotic expansion (29) follows from the standard expansion

$$
\Phi(t)=-\frac{1}{t} \varphi(t)+\frac{1}{t^{3}} \varphi(t)+O\left(t^{-5} \varphi(t)\right), \quad t \rightarrow-\infty,
$$

which holds by the integration-by-parts formula. 
Remark 2. The above proof of statement II follows the lines of the proof sketched in 2 (the proof of the implication $P_{3}(c) \Rightarrow P_{6}(c \sqrt{2})$ in Proposition 2 of [2]), where the authors referred to Beckner's lectures at the Institute Henri Poincaré. However, instead of using the equivalence

$$
I(\varepsilon) \sim \varepsilon \sqrt{2 \log \frac{1}{\varepsilon}}, \quad \varepsilon \rightarrow 0,
$$

which apparently is not sufficient to prove (28), we use stronger asymptotic expansion (29).

A more explicit condition, sufficient for the lift zonoid relation (19) to hold, can be given in a way similar to (9).

Proposition 1. There exists $c>0$ such that (19) holds if and only if there exists $\varepsilon>0$ such that

$$
\mathbf{E}_{\mu} e^{\varepsilon(v, h)_{\mathbb{R}^{d}}^{2}} \leq 2, \quad\|h\| \leq 1 .
$$

The optimal constant $c$ in (19) and $\varepsilon$ in (30) are connected by relation (10).

Since the lift zonoid order relation is equivalent to the same relation for all onedimensional projections (see [10, Section 5]), Proposition 1 follows immediately from the one-dimensional result given below.

Lemma 2. For a measure $\nu$ on $\mathbb{R}$, there exists $c>0$ such that

$$
\nu \preccurlyeq L Z \gamma_{c}
$$

with $\gamma_{c} \sim \mathcal{N}(0, c)$ if and only if there exists $\varepsilon>0$ such that

$$
\int_{\mathbb{R}} e^{\varepsilon x^{2}} d x \leq 2
$$

in that case, the optimal constants $c$ and $\varepsilon$ are connected by the relation (10).

The proof of Lemma 2 is, in fact, contained in the proof of Lemma 4.1 in [3]; hence, we omit it here.

At the end of this section, we indicate another possibility for researches related to the above results. In [4, an approach is proposed, making it possible to give explicit bounds for ergodic rates of solutions of Lévy driven stochastic differential equations, which has a wide range of further applications, e.g., to limit theorems for functionals of such processes; see [14- 16]. The key ingredient of this approach is a stochastic control based on perturbations of time coordinates of jumps of the Lévy noise. A natural question is whether such an approach remains applicable if perturbations of jump amplitudes are used instead, which is typical in the stochastic calculus of variations for processes with jumps. In this context, it would be helpful to bound from below the size of the absolutely continuous part of the image of the Lévy measure of the noise under a non-linear mapping which corresponds to the perturbation of the noise. The above results seem to be useful here, since shift inequalities yield upper bounds for the size of the singular component of the image of a measure: a respective result is obtained in 3 in the context of linear shift inequalities (6) and can be extended easily to non-linear shift inequalities (17).

\section{Weighted LOG-Sobolev Inequalities IN $\mathbb{R}$}

Theorem 2 above gives a sufficient condition for a weighted inverse log-Sobolev inequality, based on a pair of functions $v$ and $K$ related by (20). The main result of this section, Theorem 3 below, shows that the use of the same pair may lead to sufficient conditions for the (direct) log-Sobolev inequality, either in a weighted or in a classical form. What is surprising is that, even in the simplest one-dimensional case, Theorem 3 
leads to new sufficient conditions for the log-Sobolev inequality, when compared with those available in a literature; see Proposition 2 and Proposition 3 below and the two examples in Section 4. We believe that the reason for that is a proper choice of the pair of the weight functions $v$ and $K$ involved in (31) and connected by (20).

Theorem 3. Let $d=1$, and let functions $v$ and $K$ be related by (20). Assume that, for some $\alpha>0$,

$$
K v^{\prime} \geq \alpha
$$

Assume in addition that the functions $K$ and

$$
a:=2 K K^{\prime}+K^{2} v_{\mu}
$$

belong to $C^{\infty}$, have at most linear growth at $\infty$, and all their derivatives have at most polynomial growth at $\infty$.

Then

$$
\mathbf{E n t}_{\mu} f^{2} \leq \frac{2}{\alpha} \mathbf{E}_{\mu}\left(K f^{\prime}\right)^{2}
$$

for every smooth function $f$ with a compact support.

As a corollary, if $K$ is bounded, then $\mu$ satisfies the (classical) log-Sobolev inequality:

$$
\text { Ent }_{\mu} f^{2} \leq \frac{2}{\alpha}\left(\sup _{x} K^{2}(x)\right) \mathbf{E}_{\mu}\left(f^{\prime}\right)^{2}
$$

for every absolutely continuous function $f$ such that both $f$ and $f^{\prime}$ are square integrable with respect to $\mu$.

Remark 3. The proof of Theorem 3 is based on the classic Bakry-Emery criterion. We strongly believe that a similar technique is applicable in the multidimensional case as well, but because of a possible non-commutativity of matrix-valued weights which appear therein, we cannot currently give a multidimensional version of Theorem 3 , this is a subject for a further research.

Remark 4. The additional assumptions on the functions $K$ and $a$ to be smooth and to satisfy certain growth bounds can be removed, in some particular cases, by an approximation procedure; see, e.g., Propositions 2 and 3 below.

Proof of Theorem 3. Consider a Markov process $X$ defined as the strong solution to the stochastic differential equation

$$
d X_{t}=a\left(X_{t}\right) d t+\sqrt{2} K\left(X_{t}\right) d W_{t}
$$

see (32) for the definition of the coefficient $a$. Then on the Schwartz space $\mathcal{S}(\mathbb{R})$ of $C^{\infty}$ functions such that all their derivatives decay at $\infty$ faster than any polynomial, the generator $L$ of the process $X$ has the form

$$
L f=a f^{\prime}+b f^{\prime \prime}=v_{\mu} f^{\prime}+\left(b f^{\prime}\right)^{\prime}, \quad b:=K^{2} .
$$

By construction, $\mu$ is a symmetric measure for the semigroup $\left\{T_{t}\right\}$ generated by the process $X$ :

$$
\mathbf{E}_{\mu} f T_{t} g=\mathbf{E}_{\mu} g T_{t} f, \quad t \geq 0
$$

in particular,

$$
\mathbf{E}_{\mu} T_{t} f=\mathbf{E}_{\mu} f, \quad t \geq 0,
$$

i.e., $\mu$ is an invariant measure for $X$. The class $\mathcal{G}=\mathcal{S}(\mathbb{R})$ is an algebra, invariant with respect to the superpositions with $C^{\infty}$-functions and dense in every $L_{p}(\mu), p \geq 1$. In addition, in view of the smoothness conditions and growth bounds imposed on the 
coefficients $a$ and $K$, the class $\mathcal{G}$ is invariant with respect to the semigroup $T_{t}$ and the generator $L$. Define for $f, g \in \mathcal{G}$

$$
\Gamma(f, g)=\frac{1}{2}(L(f g)-f L g-g L f), \quad \Gamma_{2}(f, g)=\frac{1}{2}(L \Gamma(f, g)-\Gamma(L f, g)-\Gamma(f, L g)) .
$$

We will prove that

$$
\Gamma_{2}(f, f) \geq \alpha \Gamma(f, f), \quad f \in \mathcal{G} .
$$

Then the required statement would follow from the Bakry-Emery criterion [1].

Straightforward calculations give

$$
\begin{aligned}
\Gamma(f, g)=b f^{\prime} g^{\prime}, \\
2 \Gamma_{2}(f, f)=\left(a b^{\prime}+b b^{\prime \prime}-2 a^{\prime} b\right)\left(f^{\prime}\right)^{2}-2 b b^{\prime} f^{\prime} f^{\prime \prime}+2 b^{2}\left(f^{\prime \prime}\right)^{2} \\
=\left(a b^{\prime}+b b^{\prime \prime}-2 a^{\prime} b-\frac{\left(b^{\prime}\right)^{2}}{2}\right)\left(f^{\prime}\right)^{2}+\left(\frac{b^{\prime} f^{\prime}}{\sqrt{2}}-b f^{\prime \prime} \sqrt{2}\right)^{2} \\
\geq\left(a b^{\prime}+b b^{\prime \prime}-2 a^{\prime} b-\frac{\left(b^{\prime}\right)^{2}}{2}\right)\left(f^{\prime}\right)^{2} .
\end{aligned}
$$

Hence it is sufficient to show that

$$
2 a b^{\prime}+2 b b^{\prime \prime}-4 a^{\prime} b-\left(b^{\prime}\right)^{2} \geq 4 \alpha b
$$

in order to prove (35). Recall that

$$
v=\delta_{\mu}(K)=-K v_{\mu}-K^{\prime}
$$

hence, we can express the coefficients $a$ and $b$ in terms of the functions $K$ and $v$ :

$$
a=K K^{\prime}-K v, \quad b=K^{2} .
$$

Substituting these expressions into (36), we rewrite (36) in the following form

$$
K^{3} v^{\prime} \geq \alpha K^{2}
$$

after some transformations, which are straightforward but cumbersome and therefore omitted. The latter inequality clearly holds true under (31). Hence, applying the BakryEmery criterion, we get (33) for every $f \in \mathcal{S}(\mathbb{R})$.

If $K$ is bounded, then (34) holds for every $f \in \mathcal{S}(\mathbb{R})$ as a corollary of (33). It is a standard procedure to approximate a given absolutely continuous $f$ such that

$$
f, f^{\prime} \in L_{2}(\mu)
$$

by a sequence of smooth compactly supported $f_{n}$ in such a way that $f_{n} \rightarrow f$ and $f_{n}^{\prime} \rightarrow f^{\prime}$ in $L_{2}(\mu)$; see, e.g., the proof of Corollary 2.6.10 in [6]. Passing to the limit in (34) for $f_{n}, n \geq 1$, we complete the proof.

There is a wide choice for the pair of functions $v$ and $K$ related by (20). Below we give two versions of Theorem 3 which correspond to particular choices of this pair. The first one arises when one just takes $v(x)=x-\langle\mu\rangle$,

$$
\langle\mu\rangle=\int_{\mathbb{R}} y \mu(d y) .
$$

Proposition 2. Let measure $\mu$ on $\mathbb{R}$ have the first absolute moment and a positive continuous distribution density $p_{\mu}$. Denote

$$
\bar{K}_{\mu}(x)=\frac{1}{p_{\mu}(x)} \int_{x}^{\infty}(y-\langle\mu\rangle) p_{\mu}(y) d y, \quad x \in \mathbb{R} .
$$


I. If $\inf _{x} \bar{K}_{\mu}(x)=\alpha>0$, then

$$
\text { Ent }_{\mu} f^{2} \leq \frac{2}{\alpha} \mathbf{E}_{\mu}\left(\bar{K}_{\mu} f^{\prime}\right)^{2}
$$

for every smooth function $f$ with a compact support.

II. If, in addition, $\sup _{x} \bar{K}_{\mu}(x)=\beta<\infty$, then

$$
\text { Ent }_{\mu} f^{2} \leq 2 \bar{c}_{\mu} \mathbf{E}_{\mu}\left(f^{\prime}\right)^{2}
$$

for every absolutely continuous $f$ such that both $f$ and $f^{\prime}$ are square integrable with respect to $\mu$, where

$$
\bar{c}_{\mu}=\frac{\beta^{2}}{\alpha} .
$$

In the second version of Theorem $[3$, we choose $K$ in a more intrinsic way, namely, we take $K$ such that $\delta_{\mu}(K)=v$ with

$$
v=\Phi^{-1}\left(F_{\mu}\right), \quad F_{\mu}(x)=\mu((-\infty, x]),
$$

then $\mu \circ v^{-1}=\gamma, \gamma \sim \mathcal{N}(0,1)$. Such a choice of the weight $v$ is motivated by our intent to have

$$
\hat{Z}^{v}(\mu)=\hat{Z}(\gamma)
$$

that is, to make the order condition (19) with $c=1$ as precise as it is possible, i.e., to replace an inequality by an identity. Since $\hat{Z}^{v}(\mu)=Z\left(\mu \circ v^{-1}\right)$ identifies the law of $\mu \circ v^{-1}$ uniquely, such an intent naturally leads to the formula (37).

Proposition 3. Let a measure $\mu$ on $\mathbb{R}$ have a positive continuous distribution density $p_{\mu}$. Denote

$$
\hat{K}_{\mu}(x)=\frac{I\left(F_{\mu}(x)\right)}{p_{\mu}(x)} .
$$

I. For every smooth $f$ with a compact support,

$$
\mathbf{E n t}_{\mu} f^{2} \leq 2 \mathbf{E}_{\mu}\left(\hat{K}_{\mu} f^{\prime}\right)^{2} .
$$

II. If, in addition, $\hat{K}_{\mu}$ is bounded, then

$$
\text { Ent }_{\mu} f^{2} \leq 2 \hat{c}_{\mu} \mathbf{E}_{\mu}\left(f^{\prime}\right)^{2}
$$

for every absolutely continuous $f$ such that both $f$ and $f^{\prime}$ are square integrable with respect to $\mu$, where

$$
\hat{c}_{\mu}=\sup _{x}\left(\hat{K}_{\mu}(x)\right)^{2} .
$$

Remark 5. Define the isoperimetric function of the measure $\mu$ by

$$
I_{\mu}(p)=p_{\mu}\left(F_{\mu}^{-1}(p)\right), \quad p \in(0,1), \quad I_{\mu}(0)=I_{\mu}(1)=1 .
$$

Then, clearly, the function $I$ defined by (3) equals $I_{\gamma}, \gamma \sim \mathcal{N}(0,1)$. The function $\hat{K}_{\mu}(x)$ above can be expressed as the ratio

$$
\left.\frac{I_{\gamma}(p)}{I_{\mu}(p)}\right|_{p=F_{\mu}(x)},
$$

and the function $F_{\mu}$ gives a one-to-one correspondence between $(-\infty, \infty)$ and $(0,1)$ under the assumptions of Proposition 3 . Hence the constant $\hat{c}_{\mu}$ above can be alternatively expressed as

$$
\hat{c}_{\mu}=\left(\sup _{p \in(0,1)} \frac{I_{\gamma}(p)}{I_{\mu}(p)}\right)^{2} .
$$


Proofs of Proposition 2 and Proposition 3. If $v(x)=x-\langle\mu\rangle$, we have $\bar{K}_{\mu} v^{\prime}=\bar{K}_{\mu}$, and therefore the assumption inf $\bar{K}_{\mu}=\alpha>0$ made in Proposition 2 implies the principal condition (31). For the function $v$ defined by (37) and the function $\hat{K}_{\mu}$, this condition takes even a more simple form since straightforward calculation shows that

$$
\hat{K}_{\mu} v^{\prime}=1 \text {. }
$$

Hence one can expect that Proposition 2 and Proposition 3 follow from the version of the Bakry-Emery criterion given in Theorem 3 . However, we cannot apply this theorem here directly because of extra smoothness and growth conditions on functions $K$ and $a$ that are imposed therein. The idea of the proof is as follows: First, we consider a family of measures, which approximate $\mu$ properly and satisfy both (31) for the respective pair of $K$ and $v$, and extra smoothness and growth conditions on respective functions $K$ and $a$. Then, by passing to a limit, we get respective weighted log-Sobolev inequality, i.e., prove statements I in Propositions 2 and 3 Finally, using the same approximation procedure as in the proof of Theorem 3 above, we extend the class of functions $f$ in the case where the weight $K$ is bounded.

To shorten the exposition, we explain in details the way this strategy is implemented for the proof of Proposition [3, only. The detailed proof of Proposition 2 is similar and omitted. We also do not repeat the approximation arguments from the proof of Theorem 3 above, and concentrate on the proof of (38) for smooth compactly supported functions $f$.

First we consider the following auxiliary case: $p_{\mu} \in C^{\infty}$ is such that

$$
p_{\mu}(x)=\varphi(x), \quad|x| \geq R,
$$

for some $R>0$. Then $v_{\mu}$ (recall $\left.v_{\mu}=p_{\mu}^{\prime} / p_{\mu}\right)$ and $\hat{K}_{\mu}$ belong to $C^{\infty}$ and

$$
v_{\mu}(x)=-x, \quad \hat{K}_{\mu}(x)=1, \quad|x| \geq R .
$$

Thus the functions $K=\hat{K}_{\mu}$ and $a$ defined by (32) satisfy the assumptions of Theorem 3 . Hence, applying Theorem 3, we get (38).

Next, consider the general case. Fix some function $\chi \in C^{\infty}$ taking values in $[0,1]$ and such that $\chi(0)=0$ and $\chi(x)=1, x \geq 1$. Define

$$
\varphi_{r, \delta}(x)=\varphi(x)(\delta+(1-\delta) \chi(|x|+r)), \quad x \in \mathbb{R} .
$$

Every function $\varphi_{r, \delta}, r>0, \delta \geq 0$, belongs to $C^{\infty}$. Put

$$
M(r)=\int_{\mathbb{R}} \varphi_{r, 0}(x) d x .
$$

Then $M$ is a strictly decreasing function on $[0, \infty)$ and $M(0)<1$. For a given $Q>0$, consider the restriction $p_{\mu}^{Q}$ of $p_{\mu}$ to the segment $[-Q, Q]$, and assume that $Q$ is sufficiently large so that

$$
\int_{|x|>Q} p_{\mu}(x) d x<M(0) .
$$

Then for every $\delta$ small enough there exists a unique $r=r(Q, \delta)>0$ such that

$$
\int_{\mathbb{R}}\left(p_{\mu}^{Q}(x)+\varphi_{r, \delta}(x)\right) d x=1 .
$$

Take some non-negative $\psi \in C^{\infty}$, supported in $[-1,1]$ and such that $\int_{\mathbb{R}} \psi(x) d x=1$. Consider the probability measure $\mu_{Q, \varepsilon}$ with the density

$$
p_{\mu_{Q, \delta}}(x)=\frac{1}{\delta} \int_{[-\delta, \delta]} p_{\mu}^{Q}(y) \psi\left(\frac{x-y}{\delta}\right) d y+\varphi_{r, \delta}(x) .
$$


By construction, every $\mu_{Q, \delta}$ possesses the positive density of $C^{\infty}$ and satisfies (39) for some large $R$. Therefore, (38) holds with $\mu_{Q, \delta}$ instead of $\mu$. It can easily be seen that

$$
p_{\mu_{Q}, \delta} \rightarrow p_{\mu}, \quad K_{\mu_{Q}, \delta} \rightarrow K_{\mu}, \quad \delta \rightarrow 0, Q \rightarrow \infty
$$

uniformly on every finite segment. Passing to the limit, we obtain (38) for the initial measure $\mu$ and arbitrary smooth and compactly supported function $f$.

\section{EXAMPLES}

Example 1 . Let $\mu$ on $\mathbb{R}$ have the positive $C^{1}$-density $p_{\mu}$ such that

$$
v_{\mu}(x) x \geq-a x^{2}, \quad|x|>R,
$$

for some $a, R>0$. Our aim is to show that condition inf $\bar{K}_{\mu}>0$ of Proposition 2 holds. Changing the variables $x \mapsto x-\langle\mu\rangle$, we can restrict ourselves to the case of $\langle\mu\rangle=0$. Then we have for $x>R$

$$
\begin{aligned}
\bar{K}_{\mu}(x) & =\int_{x}^{\infty} y \exp \left(\log p_{\mu}(y)-\log p_{\mu}(x)\right) d y \\
& =\int_{x}^{\infty} y \exp \left(\int_{x}^{y} v_{\mu}(z) d z\right) d y \geq \int_{x}^{\infty} y \exp \left(-a \int_{x}^{y} z d z\right) d y \\
& =e^{a x^{2} / 2} \int_{x}^{\infty} y e^{-a y^{2} / 2} d y=1 / a
\end{aligned}
$$

A similar relation holds for $x<-R$, as well. To prove this, note that

$$
\bar{K}_{\mu}=-\frac{1}{p_{\mu}(x)} \int_{-\infty}^{x} y p_{\mu}(y) d y,
$$

since $\mu$ is centered. Finally, since $p_{\mu} \in C^{1}$ is positive, $\inf _{[-R, R]} \bar{K}_{\mu}>0$, and this completes the proof.

In addition, if

$$
v_{\mu}(x) x \leq-b x^{2}, \quad|x|>R,
$$

for some $b>0$, then $\sup \bar{K}_{\mu}<\infty$. Hence, by statement II of Proposition 2 the logSobolev inequality holds for a measure $\mu$ satisfying (40) and (41).

Note that (41) is just the well-known drift condition, sufficient for the Poincare inequality; see, e.g., [8, Theorem 3.1 and Remark 3.2]. Various sufficient conditions for the log-Sobolev inequality known in the literature require some additional assumptions on the curvature, which in the current context equals $-v_{\mu}^{\prime}$. For example, the famous Bakry-Emery condition (see [1]) requires $-v_{\mu}^{\prime} \geq \delta>0$; conditions by Wang ([17]) and Cattiaux and Guillin [8, Theorem 5.1]) are more flexible, but still contain a requirement that the curvature is bounded from below. In our case, this reduces to

$$
-v_{\mu}^{\prime} \geq \delta
$$

with some $\delta \in \mathbb{R}$. The above condition (40) can be understood as an integral version of (42), and it is easy to give an example of a measure $\mu$ satisfying (40) and (41) such that (42) fails.

Example 2. Let $\gamma^{3}$ be a standard Gaussian measure on $\mathbb{R}^{3}$, and let $B_{R}$ be a ball of radius $R$ touching the origin and with the center located at the first basis vector $e_{1}$; that is $B_{R}=B\left(R e_{1}, R\right)$. Denote by $\gamma^{3, R}$ the measure $\gamma^{3}$ conditioned outside the ball $B_{R}$ :

$$
\gamma^{3, R}(A)=\frac{\gamma^{3}\left(A \backslash B_{R}\right)}{\gamma^{3}\left(\mathbb{R}^{3} \backslash B_{R}\right)} .
$$


Consider a measure $\mu_{R}$ on $\mathbb{R}$ which is a projection of $\gamma^{3, R}$ on the first coordinate. We will show that there exists some constant $\hat{c}$ such that the constants $\hat{c}_{\mu}$ for the measures $\mu=\mu_{R}$ involved in Proposition 3 are dominated by $\hat{c}$ uniformly with respect to $R \geq 0$. This yields that the log-Sobolev inequality holds with uniformly bounded constants for the family $\mu_{R}, R \geq 0$.

For a given $x \in[0,2 R]$, the section of the ball $B_{R}$ by the hyperplane

$$
\left\{y=\left(y_{1}, y_{2}, y_{3}\right): y_{1}=x\right\},
$$

projected on the last two coordinates, is the ball in $\mathbb{R}^{2}$, centered at the origin and having the radius

$$
r_{R}(x)=\sqrt{2 R x-x^{2}} .
$$

Define $r(x)=0$ for $x \notin[0,2 R]$. Then we have for $\mu=\mu_{R}$

$$
p_{\mu}(x)=C_{R} \varphi(x) \psi_{2}\left(r_{R}(x)\right),
$$

where

$$
\begin{gathered}
C_{R}=\left(\gamma^{3}\left(\mathbb{R}^{3} \backslash B_{R}\right)\right)^{-1} \\
\psi_{2}(r)=\int_{\|y\| \geq r} \frac{1}{2 \pi} e^{-\left(y_{1}^{2}+y_{2}^{2}\right) / 2} d y_{1} d y_{2}=\frac{1}{2 \pi} \int_{0}^{2 \pi} \int_{r}^{\infty} e^{-\rho^{2} / 2} \rho d \rho d \theta=e^{-r^{2} / 2} .
\end{gathered}
$$

Consequently,

$$
p_{\mu}(x)=\frac{C_{R}}{\sqrt{2 \pi}} \begin{cases}e^{-R x}, & x \in[0,2 R] \\ e^{-x^{2} / 2}, & \text { otherwise. }\end{cases}
$$

To bound $\hat{K}_{\mu}(x)$ consider separately three cases.

I. $x<0$. Recall that $I^{\prime}(p)=-\Phi^{-1}(p)$. Then

$$
[I(c \Phi(x))]^{\prime}=-\Phi^{-1}(c \Phi(x)) c \varphi(x) \leq(-x) c \varphi(x)=c \varphi^{\prime}(x)
$$

for any $c>1$, since $\Phi^{-1}$ is an increasing function. Clearly, both $I(c \Phi(x))$ and $\varphi(x)$ vanish as $x \rightarrow-\infty$; hence,

$$
I(c \Phi(x))=\int_{-\infty}^{x}[I(c \Phi(y))]^{\prime} d y \leq c \int_{-\infty}^{x} \varphi^{\prime}(y) d y=c \varphi(x), \quad x \leq \Phi^{-1}(1 / c) .
$$

Note that

$$
F_{\mu}(x)=C_{R} \Phi(x), \quad p_{\mu}(x)=C_{R} \varphi(x),
$$

for $x<0$ and that $C_{R}>1$. In addition, the half-space $\left\{y=\left(y_{1}, y_{2}, y_{3}\right): y_{1} \leq x\right\}$ is contained in $\mathbb{R}^{3} \backslash B_{R}$, hence

$$
\Phi(x)=\gamma^{3}\left(\left\{y=\left(y_{1}, y_{2}, y_{3}\right): y_{1} \leq x\right\}\right) \leq \frac{1}{C_{R}} \Leftrightarrow x \leq \Phi^{-1}\left(\frac{1}{C_{R}}\right),
$$

and we can apply (44) to get

$$
\hat{K}_{\mu}(x)=\frac{I\left(C_{R} \Phi(x)\right)}{C_{R} \varphi(x)} \leq 1, \quad x<0 .
$$

II. $x>2 R$. In this case, $1-F_{\mu}(x)=C_{R}(1-\Phi(x))$. Recall that $I(p)=I(1-p)$ and $\Phi^{-1}(1-\Phi(x))=-x$, hence we can use the same argument as in the case $\mathbf{I}$ to show that $\hat{K}_{\mu}(x) \leq 1$, since

$$
I\left(c(1-\Phi(x))=-c \int_{x}^{\infty} \Phi^{-1}(c(1-\Phi(y))) d y \leq c \int_{x}^{\infty} y \varphi^{\prime}(y) d y=c \varphi(x)\right.
$$

for any $c>1$. 
III. $x \in[0,2 R]$. Recall that there exists a constant $c_{*}$ such that

$$
I(p) \leq c_{*} p \sqrt{\log \frac{1}{p}}, \quad p \in\left(0, \frac{1}{2}\right) .
$$

Thus

$$
C_{R} \gamma^{3}\left(\left\{y=\left(y_{1}, y_{2}, y_{3}\right): y_{1}>R\right\}\right) \leq 1-F_{\mu}(x) \leq C_{R} \gamma^{3}\left(\left\{y=\left(y_{1}, y_{2}, y_{3}\right): y_{1}>0\right\}\right)<\frac{1}{2},
$$

whence, using the identity $I(p)=I(1-p)$,

$$
\hat{K}_{\mu}(x)=\frac{I\left(1-F_{\mu}(x)\right)}{p_{\mu}(x)} \leq c_{*} \frac{1-F_{\mu}(x)}{p_{\mu}(x)} \sqrt{\log \frac{1}{1-F_{\mu}(x)}} .
$$

Since $C_{R}>1$, we have

$$
\log \frac{1}{1-F_{\mu}(x)} \leq \log \frac{1}{1-F_{\mu}(2 R)}=\log \frac{1}{C_{R}(1-\Phi(2 R))} \leq \log \frac{1}{1-\Phi(2 R)} \leq c^{*}(1+R)^{2}
$$

with some $c^{*}>2$. By (43), we have

$$
\frac{1-F_{\mu}(x)}{p_{\mu}(x)}=e^{R x}\left(\int_{x}^{2 R} e^{-R y} d y+\int_{2 R} e^{-y^{2} / 2} d y\right),
$$

and the right-hand side can be estimated either by

$$
e^{R x} \int_{x}^{\infty} e^{-R y} d y=\frac{1}{R}
$$

(if $R$ is large), or by

$$
e^{2 R^{2}} \int_{0} e^{-y^{2} / 2} d y=\sqrt{\frac{\pi}{2}} e^{2 R^{2}}
$$

(if $R$ is small). Then

$$
\hat{c}_{\mu}=\sup _{x} \hat{K}_{\mu} \leq \hat{c}:=c_{*} c^{*} \sup _{Q>0} \min \left(\frac{1+Q}{Q}, \sqrt{\frac{\pi}{2}}(1+Q) e^{2 Q^{2}}\right)
$$

for $\mu=\mu_{R}$ and for any $R>0$. If $R=0$, then the measure $\mu$ equals $\gamma$ and therefore $\hat{c}_{\mu}=1$.

This example is motivated by the manuscript [5], where the problem of estimating the Poincaré constant is considered for a Gaussian measure conditioned outside a ball. An approach proposed therein is based on the decomposition of the variance and requires an estimate for the Poincaré constant of the one-dimensional projection of the "punctured" Gaussian measure to the line which contains the center of the ball. Such an estimate depends on the position and size of the ball; see [5, Lemma 4.7]. The case of a large ball touching the origin relates the case (4) of Lemma 4.7 in [5]. Our estimate for the log-Sobolev constant implies that the Poincaré constant for $\mu$ is uniformly bounded by $\hat{c}$, which improves essentially the bound $c e^{R^{2}}$ obtained in [5, Lemma 4.7, statement (4)]. Heuristically, the reason for this is the following. The measure $\mu$ contains "cavities", which appear due to the puncturing procedure. If the ball is "large" and is located not "far from the origin", then these cavities make the bounds for the Poincaré inequality obtained via classic sufficient conditions to be very inaccurate. On the other hand, the form of the weight $\hat{K}_{\mu}$ in Proposition 3 is highly adjusted to these cavities which makes the corresponding bounds more precise. We believe that similar calculations can be made in a general setting, that is for an arbitrary $d \geq 2$ and arbitrary position and size of the ball; this is a subject of a further research. 


\section{ACKNOWLEDGMENTS}

The paper was prepared partially during a visit of the authors to the University of Potsdam; the authors express their deep gratitude to the University of Potsdam and personally to Sylvie Roelly for their hospitality. The authors are also grateful to Emmanuel Boissard for fruitful discussions concerning the manuscript [5].

\section{BIBLIOGRAPHY}

1. D. Bakry and M. Emery, Hypercontractivity for diffusion semi-groups, Comptes rendus des seances de l'Academie des sciences. Serie 1299 (1984), no. 15, 775-778. MR772092(86f:60097)

2. F. Barthe, D. Cordero-Erausquin, and M. Fradelizi, Gaussian shift inequalities and norms of barycenters, Studia Math. 146 (2001), 245-259. MR.1853445(2002h:28004)

3. S. G. Bobkov, The size of singular component and shift inequalities, Ann. Probab. 27 (1999), no. 1, 416-431. MR1681090 (2000e:60025)

4. S. V. Bodnarchuk and A. M. Kulik, Stochastic control based on time-change transformations for stochastic processes with Lévy noise, Probab. Theor. Math. Statist. 86 (2012), 11-27.

5. E. Boissard, P. Cattiaux, A. Guillin, and L. Miclo, Ornstein-Uhlenbeck Pinball: I. Poincaré Inequalities in a Punctured Domain, Manuscript.

6. V. I. Bogachev, Differentiable Measures and the Malliavin Calculus, AMS, Providence, 2010. MR2663405 (2012c:60146)

7. I. Cascos, Data depth: multivariate statistics and geometry, New Perspectives in Stochastic Geometry (W. S. Kendall and I. Molchanov, eds.), Oxford Univ. Press, Oxford, 2010, pp. 398426. MR2654685 (2011e:62003)

8. P. Cattiaux and A. Guillin, Functional inequalities via Lyapunov conditions, arXiv:1001.1822.

9. G. A. Koshevoy and K. Mosler, Zonoid trimming for multivariate distributions, Ann. Stat. 25:5 (1997), 1998-2017. MR 1474078 (99c:62151)

10. G. A. Koshevoy and K. Mosler, Lift zonoids, random convex hulls and the variability of random vectors, Bernoulli 4 (1998), 377-399. MR1653276 (99k:60021)

11. A. M. Kulik and T. D. Tymoshkevych, Lift zonoid and barycentric representation on a Banach space with a cylinder measure, Lithuanian Math. J. 53 (2013), no. 2, 181-195. MR3265745

12. I. Molchanov and M. Schmutz, Exchangeability type properties of asset prices, Adv. Appl. Probab. 43 (2011), 666-687. MR2858216

13. I. Molchanov, M. Schmutz, and K. Stucki, Invariance properties of random vectors and stochastic processes based on the zonoid concept, arXiv:1203.6085. MR.3217442

14. A. Yu. Veretennikov and A. M. Kulik, On extended Poisson equation for weakly ergodic Markov processes, Probab. Theor. Math. Statist. 85 (2011), 22-38. MR2933700 (2012m:60128)

15. A. Yu. Veretennikov and A. M. Kulik, Diffusion approximation for systems with weakly ergodic Markov perturbations I, Probab. Theor. Math. Statist. 87 (2012), 1-16. MR3112631

16. A. Yu. Veretennikov and A. M. Kulik, Diffusion approximation for systems with weakly ergodic Markov perturbations II, Probab. Theor. Math. Statist. 88 (2013), 1-16. MR3112631

17. F. Y. Wang, Functional inequalities for empty essential spectrum, J. Funct. Anal. 170 (2000), no. 1, 219-245. MR1736202(2001a:58043)

Institute of Mathematics, National Academy of Science of Ukraine, Tereshchenkivska Street 3, 01601, Kyiv, Ukraine

E-mail address: kulik.alex.m@gmail.com

National Taras Shevchenko University of Kyiv, Academician Glushkov Avenue 4-e, 03127, Kyiv, Ukraine

E-mail address: tymoshkevych@gmail.com

Received 4/SEP/2013

Originally published in English 